\title{
Unplanned 30-Day Readmissions after Hyper-CVAD Chemotherapy in Patients with Acute Lymphoblastic Leukemia
}

\author{
Mehdi Dehghani $i^{1, *}$, Shirin Haghighat ${ }^{2}$, Zahra Radmard ${ }^{3}$, Nasrin Namdari ${ }^{2}$, Alireza Rezvani ${ }^{1}$ \\ and Mani Ramzi ${ }^{1}$
}

${ }^{1}$ Hematology Research Center, Department of Hematology and Medical Oncology, Shiraz University of Medical Sciences, Shiraz, Iran

${ }^{2}$ Department of Hematology and Medical Oncology, Shiraz University of Medical Sciences, Shiraz, Iran

${ }^{3}$ Department of Internal Medicine, Shiraz University of Medical Sciences, Shiraz, Iran

\begin{abstract}
Introduction: The aim of this study was to investigate the rate and causes of re-hospitalization in the first 30 days after Hyper-CVAD chemotherapy in all patients with acute lymphoblastic leukemia.

Methods: This descriptive, analytical and cross-sectional study was performed on 827 admissions in ALL patients aged 18 years and older with unplanned hospitalization after HyperCVAD chemotherapy in less than 30 days' post chemotherapy from April 2016 to April 2018 in Hematology and medical oncology department.

Results: The rate of unplanned re-admission was $9.91 \%$ in all patients. Mean follow-up time was $13.77 \pm 6.26$ months and the mean age of patients was $35.55 \pm 14.6$ years. Re-admission rate was more frequent in men $(65.7 \%)$ and most patients were readmitted only once. The mean duration of re-admission was $8.2 \pm 4.15$ days and most patients were readmitted after cycles IB, IIIB and IVB of hyper-CVAD chemotherapy. The most common causes of readmission were febrile neutropenia and pancytopenia. Except for significant changes in CBC, no significant changes were observed in other laboratory tests. Urine culture and blood culture were reported positive in $13.6 \%$ and $31.57 \%$ respectively, and Ecoli was the most common organism isolated from cultures.

Conclusion: We found increased rate of re-admission following hyper-CVAD chemotherapy in patients with acute lymphoblastic leukemia which was due to side effects of chemotherapy regimen. It seems to be important not only for high rate of mortality and morbidity in patients resulting from this chemotherapy regimen, but also imposing the heavy cost on health system. Therefore, more effective preventive measures are necessary and useful.
\end{abstract}

Keywords: Acute lymphocytic leukemia, Unplanned, Readmission, Hyper-CVAD, Chemotherapy.

\section{INTRODUCTION}

Cancer treatment is becoming increasingly complex and costly, raising awareness among patients, physicians, health care personnel and insurance systems. A goal in cost-effective care as well as focusing on joint decision-making can help us to improve quality and reduce cancer care costs [1]. Cancer patients are often hospitalized due to unpredictable conditions or resistant symptoms. The main complaints on admission are shortness of breath, pain and nervous system problems. Although, most hospitalizations lead to discharge from the hospital, but re-admission and death have been reported in some patients with advanced disease in less than thirty days after discharge [2].

In a retrospective cohort study on elderly patients with cancer, the 30 days unplanned readmission rate was reported $35.2 \%$. The most common reasons for readmission were cardiovascular, pulmonary disease, failure to thrive and neurological disease [3]. There are

*Address correspondence to this author at the Hematology Research Center, Department of Hematology and Medical Oncology, Shiraz University of Medical Sciences, Shiraz, Iran; E-mail: mehdi_dehghani6@yahoo.com many factors involved in the re-admission of patients with cancer, especially malignant hematologic disorders. Abnormal laboratory tests such as hemoglobin, albumin, sodium and SGOT at discharge are found to be important predictors for unexpected admission in elderly patients with cancer [4]. The others risk factors for unplanned re-hospitalization are severity of illness, recurrence or metastasis of the malignancy and the presence of three or more co-morbidities [5].

Finding ways to reduce the rate of unplanned rehospitalization remains an important concern in the field of oncology. This goal can be achieved by identifying the facilitating factors accurately. The age and general performance level of the patient; presence of any co-morbid disease; and the stage of the malignant disease are among these factors. Other living conditions also matter; for example, according to the studies patients who had been discharged or admitted to hospice were less likely at risk of readmission $[5,6]$.

\section{MATERIAL AND METHOD}

This is a descriptive, analytical, cross-sectional study of 827 hospitalizations and 452 re-admissions, in 
which 82 patients had unplanned hospitalization after HyperCVAD chemotherapy within 30 days' postchemotherapy. The study period was from April 2016 to April 2018 in the Department of Hematology and Medical Oncology of Amir Hospital and the approval of this project has been provided by Shiraz University of Medical Sciences (SUMS).

\section{Inclusion Criteria}

Patients were selected from the beginning of the study by census method, which included all patients diagnosed with acute lymphocytic leukemia at least once unplanned readmission to the tertiary Cancer Hospital within 30 days after initial hospitalization. All patients 18 years of age or older who were readmitted less than one month after hyperCVAD chemotherapy due to possible chemotherapy complications or disease recurrence were selected.

\section{Exclusion Criteria}

Patients under 18 years of age, those scheduled to be hospitalized for the next cycle of chemotherapy, those whose records were unavailable for any reason, those who were admitted for reasons unrelated to chemotherapy, such as Port embedded, those hospitalized under 6 hours of outpatient admission for blood products and supportive treatment, those who were hospitalized with recurrent disease after 30 days, who had recurrent relapses and others who were sent from other centers. Scheduled admissions, such as selective chemotherapy or inpatient chemotherapy, as well as unrelated hospitalizations such as hospitalizations for diagnostic work up or minor surgeries unrelated to previous chemotherapy including port catheter insertion were excluded.

Data were collected by using a questionnaire. Questionnaire information included age, gender, any underlying disease (diabetes, hypertension, renal disease, cardiac disease, etc.); date of diagnosis of ALL; the time interval between readmission and the last course of chemotherapy; and the mean readmission time. Also, the biochemical blood profile of the patients at re-admission and after chemotherapy, culture results, causes of readmission, presence or absence of bleeding, re-admission outcome and final outcome were examined at the end of the study. Descriptive statistics were applied to summarize demographic and clinical data. $P$ values were considered significant at the 0.05 level. Data were analyzed using descriptive and inferential statistics using SPSS V.21 software.

\section{Ethics Statements}

In this study, all steps related to patient participation were performed in accordance with the ethical standards of the Committee for Research and Communication and Helsinki Declaration 1964 and subsequent amendments as well as comparable ethical standards. This study was approved by the ethics committee of Shiraz University of Medical Sciences, Shiraz, Iran (code: IR. sums. med. rec. 1397.325) Due to the retrospective nature of the study, formal consent was not required.

\section{RESULTS}

In this study, out of a total of 827 hospitalizations, 452 patients were re-hospitalized under 30 days, of which only 82 patients had unplanned re-admission who met the criteria for enrolling to the study. Overall, hospitalization less than 30 days was $54.5 \%$ and rate of unplanned readmission in these patients was $9.9 \%$. There were no cases of relapse or refractory disease at unplanned 30-Day readmissions after Hyper-CVAD chemotherapy.

In this cross-sectional study median age of patients were $35.5 \pm 14.6$ (ranged 18-75) and they were followed for median $13.7 \pm 6.2$ months. Most patients were rehospitalized once (69.5\%). Twice re-hospitalization and three re-hospitalizations and four re-hospitalizations were $15.9 \%, 7.3 \%$ and $6.1 \%$, respectively. One patient had six unplanned re-hospitalizations, which appears to be due to his advanced age, which was 75 years old.

According to the Table 1, the most common comorbidities were hypertension, diabetes, cardiovascular disease and central nervous system disease in that order. Of all patients, one had rheumatoid arthritis, one had pulmonary disease, and one had a history of aspergillus coinfection. The mean hospital stay for hyperCVAD chemotherapy was $5.8 \pm$ 3.3 days. Most patients were hospitalized for more than or equal to 5 days for chemotherapy (72\%). The average duration of unplanned re-hospitalization after chemotherapy is $8.2 \pm 4.1$ days and most patients were re-hospitalized after cycles IIIB, IVB and IB of hyperCVAD chemotherapy. Therefore, we need to be more careful in these treatment cycles for re-admission especially for febrile neutropenia and pancytopenia, because these two causes are the most common cause of re-hospitalization in these patients.

After receiving chemotherapy, $64.6 \%$ and $25.6 \%$ of patients received GGF and PEGylated GCSF, 
Table 1: Frequency of Re-Hospitalization Based on Underlying Disease

\begin{tabular}{|c|c|}
\hline Underlying disease & Percentage \\
\hline \hline Cardiac & $4.9 \%$ \\
\hline Hypertension & $9.8 \%$ \\
\hline Diabetes & $8.5 \%$ \\
\hline Deep vein thrombosis & $6.1 \%$ \\
\hline Central nervous system involvement & $7.4 \%$ \\
\hline seizure & $1.2 \%$ \\
\hline Second primary cancer & $3.6 \%$ \\
\hline
\end{tabular}

respectively, but about $10 \%$ of patients did not receive any growth factor after discharge from the hospital. The mean number of GCSF injected was $2.87 \pm 0.11$ and in the case of PEGylated GCSF injection, it was $1.05 \pm 0$. 048. According to Table 2, the most common causes of unplanned hospitalization are fever, neutropenia, pancytopenia, pneumonia, and perianal abscess. The most common hematologic disorders after hperCVAD chemotherapy were pancytopenia $(92.7 \%)$, bicytopenia $(3.7 \%)$ and isolated anemia (2.4\%); although isolated thrombocytopenia was seen in only one patient.

Table 2: Causes and Frequency of Re-Hospitalization after Chemotherapy

\begin{tabular}{|c|c|}
\hline Underlying disease & Percentage \\
\hline \hline Neutropenic fever & $49.9 \%$ \\
\hline Sever pancytopenia & $41.5 \%$ \\
\hline Pneumonia & $2.4 \%$ \\
\hline Perianal abscess & $2.4 \%$ \\
\hline Diarrhea & $1.2 \%$ \\
\hline Deep vein thrombosis & $1.2 \%$ \\
\hline Facial palsy & $1.2 \%$ \\
\hline Total & $100 \%$ \\
\hline
\end{tabular}

A large number of patients had a normal white cell count $(68.3 \%)$ after receiving chemotherapy at the time of discharge from hospital, while in re-hospitalization, most patients had grade 4 neutropenia (51.2\%). About $(13.3 \%)$ of patients referred with hemorrhage and the most common of which is nosebleeds, followed by vaginal bleeding in women. Other bleeding sites include bleeding from the oral cavity, gastrointestinal bleeding, hemoptysis, and pulmonary hemorrhage. The minimum count of WBCs in re-hospitalization was $100 /$ dl with average of $1029.8 \pm 1648.7$. The minimum WBC that patients had at the time of discharge after chemotherapy was $200 / \mathrm{dl}$.

The lowest and mean hemoglobin levels of patients at the time of readmission after chemotherapy were 4.1 $\mathrm{mg} / \mathrm{dl}$ and $7.99 \pm 1.59$ respectively. In the case of electrolytic disturbances, hyponatremia and hypokalemia were seen both in re-hospitalization and after discharge. The maximum BUN of patients was 61 $\mathrm{mg} / \mathrm{dl}$ and the mean creatinine level was almost normal $(0.18 \pm 0.87 \mathrm{mg} / \mathrm{dl})$, some patients had some degree of renal insufficiency (maximum creatinine levels of 2.1 $\mathrm{mg} / \mathrm{dl}$ in readmission and $1.4 \mathrm{mg} / \mathrm{dl}$ post chemotherapy). No significant abnormalities were seen in the mean liver function tests, although hypoalbuminemia was evident (minimum albumin levels of $2.8 \mathrm{mg} / \mathrm{dl}$ in readmission and $3 \mathrm{mg} / \mathrm{dl}$ post chemotherapy). The mean body mass index was 23.98 $\pm 4.97 \mathrm{Kg} / \mathrm{m}^{2}$ and the highest percentage was related to the patients with normal range of body mass index $(43.9 \%)$.

The end result of the study was defined as death and survival, most patients (79 patients) survived in unplanned hospitalization (96.3\%) and 3 patients were died $(3.7 \%)$. The frequency of positive urine culture results in re-hospitalization was $13.16 \%$ and the most common organisms isolated from urine cultures included gram-negatives including $\mathrm{E}$-coli and Klebsiella.

The high percentage of positive blood culture results was $14.9 \%$. The most common organisms isolated from the blood culture included gram-negatives including E-Coli and Klebsiella and gram-positive organisms such as DNAase coagulase Negative staphylococci. The results of other patient cultures (port-a-catheter and double lumen cultures) were reported to be $16.67 \%$. In total, the blood culture and port-a-catheter were positive $31.77 \%$.

\section{DISCUSSION}

Annually large sums of the health system budget are spent on planned and unplanned hospitalizations for treatment and side effects or complications of therapy. Unfortunately, the incidence of malignancies is increasing day by day in the world, and mortality due to cancer is one of the major leading causes of death in the world [7]. It is possible that hematologic malignancies, especially ALL, are no exception to this rule. To gain further knowledge about the beneficial and side effects of chemotherapeutic agents in 
individual patients seems rational to optimally prevent the re- admission.

In this study, the percentage of unplanned rehospitalization in less than 30 days was $9.9 \%$. Percentage of unplanned readmission in a study by Tran et al. were $(22.4 \%)$. The top three causes for unplanned readmissions were neutropenia/ agranulocytosis $(27.8 \%)$, septicemia $(15.3 \%)$, and pancytopenia (11.5\%) [8]. unplanned readmission after autologous and allogenic hematopoietic cell transplants were $11.6 \%$ and $24.4 \%$, respectively in a study by Dhakal et al. Although similar studies have not been found in patients with acute leukemia after chemotherapy, this is lower than the study by Dhakal et al. on unplanned readmission after autologous and allogenic hematopoietic cell transplants that were $11.6 \%$ and $24.4 \%$, respectively. The most common reasons for re-hospitalization included infection, gastrointestinal complains and hospital stem cell transplantation volume that was inversely associated with readmission rate [9]. If Compared the readmission rate to the study by $\mathrm{H}$. Hoyer $(20 \%)$ and Spring et al. $(21 \%)$, it is surprisingly lower $[10,11]$.

In most studies around the world, comorbidities have been studied as an important risk factor for unplanned hospitalizations. Underlying disease including diabetes, chronic lung disease, renal failure, cardiac disease and cancer is an important factor for re hospitalization in all patients in internal medicine wards $[12,13]$.

Regardless of the underlying disease, the number of which is two, three, or more, as well as metastatic disease has been associated with unexpected hospitalization. Literature has shown that more complex the disease, greater the chances of rehospitaliazaion [14].

In our study, high blood pressure, diabetes, and heart disease were the most common underlying diseases. Although CNS involvement is common in ALL patients, in this study the rate of CNS involvement as re-hospitalization was not high, which could be due to the high dose of methotrexate in the HyperCVAD chemotherapy. The most important factors for readmission in cancer patients are disease stage, discharge status and age. It makes sense for older people to be re-hospitalized with lower performance status. Additionally, patients with more advanced disease are more likely to have unpredictable hospitalization. Other important factor involved in an increased rate of re-hospitalization included the patient's health status at the time of discharging from hospital and the health at home, hospice or another facility in the following days [15].

Patients with nausea or vomiting and problems with care, or people living alone, are at higher risk for readmission within few days after discharge. Awareness of the factors that may put cancer patients at greater risk for re-hospitalization, as well as appropriate interventions during hospitalization, may help reduce the risk of re-hospitalization. It is important to provide a basis for patient evaluation, planning, intervention, and follow-up to reduce the risk of re-hospitalization [16].

In this study, the mean body mass index was 23.98 $\pm 4.97 \mathrm{Kg} / \mathrm{m}^{2}$. Most of our cases (43.9\%) were in normal weight range. The standard for unplanned rehospitalization was not investigated. However, the review article of Entezari Maleki et al. described the highest toxicity of chemotherapy drugs in patients with BMI of $18-22 \mathrm{~kg} / \mathrm{m}^{2}$ [16]. Jeremy Lund et al. reported $11.1 \%$ chemotherapy toxicity and the most important risk factors for re-hospitalization were age more than 65 years and hematological cancers, especially leukemia $[17,18]$.

The average hospital stay for chemotherapy patients was $5.83 \pm 3.3$ days. The mean re-admission time after chemotherapy is $8.2 \pm 4.15$ days. Joanna Grace Manzano and her colleagues reported a median re-admission time of 10 days or more for metastatic cancers [13]. Girish Kunapareddy also stated that the average interval from the previous discharge after chemotherapy was 11 days, which is slightly different from the present study. Doxorubicin $(7.7 \%)$ was the most widely used anthracycline. According to a study by Jeremy Lund et al. among the chemotherapeutic drugs, anthracyclines were associated with an increased risk of re-hospitalization The most common causes of hospitalization in this group were neutropenia fever, diarrhea and dehydration [17].

\section{CONCLUSION}

The need for research into the effects of chemotherapy is well felt. The valuable findings of this study show that although there are clear and valid treatment and prevention protocols in this field, and despite the fact that many of the side effects of chemotherapy are unavoidable, much of the rehospitalization is unplanned. There are preventable causes, so paying more attention to this issue can help 
reduce unwanted hospitalizations and improve the quality of life and survival of patients, and prevent the imposition of huge costs on the health system for reasons that are easily preventable.

\section{FUNDING/SUPPORT}

This Study was supported by Shiraz University of Medical Sciences

\section{CONFLICT OF INTEREST}

None disclosed.

\section{REFERENCES}

[1] Kumar P, Moy B. The Cost of Cancer Care-Balancing Our Duties to Patients Versus Society: Are They Mutually Exclusive? The Oncologist 2013; 18(4): 347. https://doi.org/10.1634/theoncologist.2013-0078

[2] Numico G, Cristofano A, Mozzicafreddo A, Cursio OE, Franco $\mathrm{P}$, Courthod $\mathrm{G}$, et al. Hospital admission of cancer patients: avoidable practice or necessary care? PloS One 2015; 10(3). https://doi.org/10.1371/journal.pone.0120827

[3] Chiang LY, Liu J, Flood KL, Carroll MB, Piccirillo JF, Stark S, et al. Geriatric assessment as predictors of hospital readmission in older adults with cancer. Journal of Geriatric Oncology 2015; 6(4): 254-61.

https://doi.org/10.1016/j.jgo.2015.04.003

[4] Burhenn P, Sun C-L, Scher KS, Hsu J, Pandya P, Chui C-Y, et al. Predictors of hospital readmission among older adults with cancer. Journal of Geriatric Oncology 2020. https://doi.org/10.1016/j.jgo.2020.03.008

[5] Zhou H, Roberts PA, Dhaliwal SS, Della PR. Risk factors associated with paediatric unplanned hospital readmissions: a systematic review. BMJ Open 2019; 9(1): e020554. https://doi.org/10.1136/bmjopen-2017-020554

[6] Timmers T, Verhofstad M, Moons K, Leenen L. Patients' characteristics associated with readmission to a surgical intensive care unit. American Journal of Critical Care 2012; 21(6): e120-e8. https://doi.org/10.4037/ajcc2012773

[7] Farhood B, Geraily G, AlizadehA. Incidence and Mortality of Various Cancers in Iran and Compare to Other Countries: A Review Article. Iran J Public Health 2018; 47(3): 309-316.

[8] Tran PT, Slayton WB, Dalal M, Brown J. Incidence and Risk Factors for 30-Day Readmission after Inpatient Chemotherapy among Acute Lymphoblastic Leukemia Patients. Healthcare (Basel) 2020; 8(4): 401. https://doi.org/10.3390/healthcare8040401
Dhakal B, Giri S, Levin A, Rein L, Fenske TS, Chhabra S, et al. Factors associated with unplanned 30-day readmissions after hematopoietic cell transplantation among US hospitals. JAMA Network Open 2019; 2(7): e196476-e. https://doi.org/10.1001/jamanetworkopen.2019.6476

[10] Hoyer EH, Needham DM, Miller J, Deutschendorf A, Friedman M, Brotman DJ. Functional status impairment is associated with unplanned readmissions. Archives of Physical Medicine and Rehabilitation 2013; 94(10): 1951-8. https://doi.org/10.1016/j.apmr.2013.05.028

[11] Spring L, Li S, Soiffer RJ, Antin JH, Alyea III EP, Glotzbecker B. Risk factors for readmission after allogeneic hematopoietic stem cell transplantation and impact on overall survival. Biology of Blood and Marrow Transplantation 2015; 21(3): 509-16.

https://doi.org/10.1016/j.bbmt.2014.11.682

Kwok CS, Capers IV Q, Savage M, Gulati M, Potts J, Mohamed $\mathrm{MO}$, et al. Unplanned hospital readmissions after acute myocardial infarction: a nationwide analysis of rates, trends, predictors and causes in the United States between 2010 and 2014. Coronary Artery Disease 2020; 31(4): 35464.

https://doi.org/10.1097/MCA.0000000000000844

[13] Sadati AK, Lankarani KB, Tabrizi R, Rahnavard F, Zakerabasali S. Evaluation of 30-Day Unplanned Hospital Readmission in a Large Teaching Hospital in Shiraz, Iran. Shiraz E-Medical Journal 2017; 18(4). https://doi.org/10.5812/semj.39745

[14] Manzano J-GM, Gadiraju S, Hiremath A, Lin HY, Farroni J, Halm J. Unplanned 30-day readmissions in a general internal medicine hospitalist service at a comprehensive cancer center. Journal of Oncology Practice 2015; 11(5): 410-5. https://doi.org/10.1200/JOP.2014.003087

[15] Weaver C, Schiech L, Held-Warmkessel J, Kedziera P, Haney E, DiLullo G, et al., editors. Risk for unplanned hospital readmission of patients with cancer: results of a retrospective medical record review. Oncology Nursing Forum 2006.

https://doi.org/10.1188/06.ONF.E44-E52

[16] Entezari-Maleki T, Margedari S, Gholami K, Hadjibabaie M, Oghazian MB, Salamzadeh J, et al. Evaluation of chemotherapy toxicity in acute lymphoblastic and myelogenous leukemia. International Journal of HematologyOncology and Stem Cell Research 2012: 8-12.

[17] Kunapareddy G, Ahmed H, Patel SS, Patel BJ, Elson P, Bitterice A, et al. Predictors for Recurrent 30-Day Unplanned Readmissions in Patients with Hematologic Malignancies. Blood 2017; 130(Supplement 1): 532.

[18] Lund J, Pearson A, Keriazes G. Identification of Risk Factors for Chemotherapy-Related 30-Day Readmissions. Journal of the National Comprehensive Cancer Network 2015; 13(6): 748-54.

https://doi.org/10.6004/jnccn.2015.0089

(C) 2021 Dehghani et al.; Licensee Neoplasia Research.

This is an open access article licensed under the terms of the Creative Commons Attribution Non-Commercial License (http://creativecommons.org/licenses/by-nc/3.0/) which permits unrestricted, non-commercial use, distribution and reproduction in any medium, provided the work is properly cited. 